\title{
USO DE QUITINA MODIFICADA SUPORTADA EM AREIA PARA ADSORÇÃO DE CORANTE EM LEITO FIXO
}

\author{
J. M. N. DOS SANTOS ${ }^{1}$, R. DA ROSA ${ }^{1}$, G. L. DOTTO ${ }^{1 *}$ \\ ${ }^{1}$ Universidade Federal de Santa Maria, Departamento de Engenharia Química \\ *e-mail: guilherme_dotto@yahoo.com.br
}

\begin{abstract}
RESUMO
Neste estudo, a adsorção de Azul de Metileno (MB) em quitina modificada suportada por areia (quitina-SMU/areia) em leito fixo foi investigada. Para a realização dos ensaios em leito fixo, a quitina-SMU foi preparada, caracterizada e suportada em areia. As curvas de ruptura foram obtidas em diferentes vazões e com concentração inicial de $\mathrm{MB}$ igual a $50 \mathrm{mg} \mathrm{L}^{-1}$. Os dados experimentais obtidos foram ajustados a alguns modelos dinâmicos e a regeneração do leito também foi estudada. A melhor condição para a adsorção em leito fixo de MB em quitina-SMU/areia ocorreu na vazão de $10 \mathrm{~mL}$ $\min ^{-1}$. Nesta condição, o tempo de ruptura foi de $370 \mathrm{~min}$, a capacidade máxima da coluna foi $51.8 \mathrm{mg} \mathrm{g}^{-1}$ e o percentual de remoção foi $51.5 \%$. Os modelos dinâmicos foram adequados para representar os dados experimentais. A regeneração da coluna foi possível durante cinco ciclos, mantendo o mesmo desempenho do leito. Estes resultados indicaram que a adsorção, em leito fixo de MB em quitina-SMU/areia é uma técnica viável e pode ser adequada para aumento de escala.
\end{abstract}

\section{INTRODUÇÃO}

Existem aproximadamente 100.000 diferentes tipos de corantes comerciais e pigmentos. Eles são produzidos, ao redor do mundo, numa escala de $7 \times 10^{5}$ ton ao ano. Dessa produção, estima-se que $10-15 \%$ dos corantes estão presentes nos efluentes de indústrias têxteis e de alimentos (ZOLLINGER, 1992). O Azul de Metileno, um corante catiônico, é tóxico à medida que dificulta a passagem de luz através da água de corpos receptores, causando sérios impactos ambientais ao ecossistema aquático (ESQUERDO et al., 2014). De modo a evitálos, é essencial que os efluentes contaminados sejam tratados antes de serem descartados. Em geral, os principais métodos para tratar efluentes que contém corantes são adsorção, oxigenação-ozonização, tratamento biológico, coagulação-floculação e processos que utilizam membranas (WALKER et al., 2003). O tipo de processo empregado vai depender da natureza do poluente. No entanto, a adsorção é considerada a operação mais apropriada para remoção tanto de poluentes inorgânicos e orgânicos e, apresenta relativa facilidade de operação (MOURA et al., 2006).

A adsorção utilizando adsorventes de baixo custo, como a quitina, é uma alternativa eficiente e econômica para a descontaminação da água, visto que os biossorventes estão disponíveis na natureza em grande quantidade, além de serem considerados ecologicamente corretos (BHATNAGAR, SILLANPÄÄ., 2009). A quitina é um polímero natural presente nas cascas de crustáceos, estrutura de insetos e em paredes das células de fungos (KAO et al., 2009). Por sua vez, apresenta pequena área superficial 
específica, que pode significar a redução de seu potencial adsorvente. Como alternativa para melhorar esta característica, o tratamento ultrassônico é uma alternativa, pois, ao emitir ondas mecânicas, com diferentes ciclos e amplitudes, cria uma região termodinamicamente instável e bolhas no líquido que ao se romperem próximo ao material, podem causar modificações estruturais (BREITBACH, BATHEN., 2001). Os estudos no campo de adsorção de corantes em quitina, em sua maioria, são conduzidos através de experimentos em batelada (DOTTO et al., 2011). Dessa forma, é notada a importância de estudos que utilizam coluna de leito fixo, que são mais relevantes para os sistemas reais de operação (YIN et al., 2009).

Levando em consideração que as partículas de quitina com superfície modificada podem oferecer limitações ao sistema, como perda de pressão hidrostática, obstrução da coluna e formação de caminhos preferenciais (DOTTO et al., 2015). O uso de materiais para suporte, como areia, esferas de vidro e betonita, pode ser uma alternativa para solucionar esse tipo de problema (VIEIRA et al., 2014). Este trabalho teve como objetivo avaliar a adsorção do corante catiônico azul de metileno utilizando uma coluna constituída de quitina modificada suportada por areia.

\section{MATERIAIS E MÉTODOS}

\subsection{Preparação e Caracterização da Quitina com Superfície Modificada}

A quitina com superfície modificada ultrassonicamente (quitina-SMU) foi preparada de acordo com trabalhos anteriores (DOTTO et al., 2015). A quitina foi obtida a partir das cascas de camarão (Penaeus brasiliensis) através de um processo de desmineralização, desproteinização, desodorização e secagem (DOTTO et al., 2011). As amostras de quitina foram trituradas e peneiradas até que fossem obtidas partículas com diâmetro variando entre 105 e
$125 \mu \mathrm{m}$. Posteriormente, utilizando água deionizada, uma suspensão de quitina em água foi preparada e submetida ao tratamento ultrassônico (UP400S, Hieslscher, Germany) com potência de $400 \mathrm{~W}$, equipado com uma sonda de titânio, durante 1h, a $24 \mathrm{kHz}$ (o tratamento foi realizado com ciclo $1.00 \mathrm{e}$ amplitude de 60\%) (DOTTO et al., 2015). A suspensão foi filtrada e, a quitina retida, seca e armazenada para posterior uso.

As características estruturais, da quitina in natura e modificada, foram visualizadas por microscopia eletrônica de varredura (MEV) (Jeol, JSM-6610LV) (GOLDSTEIN et al., 1992). A área superficial específica foi determinada pelo método BET (Quantachrome Instruments, New Win 2) (BRUNAUER et al., 1938). Os grupos funcionais e o grau de desacetilação do adsorvente foram identificados através de (FT-IR) (Prestige, 21210045) (SILVERSTEIN et al., 2007). O índice de cristalinidade foi determinado por Difração de Raios-X (XRD) (Rigaku, Miniflex 300) (ALSAGHEER et al., 2009).

\subsection{Experimentos de Leito Fixo}

Os experimentos de leito fixo foram realizados em um sistema de laboratório, apresentado na Figura 1.

Figura 1 - Sistema de laboratório para adsorção em coluna

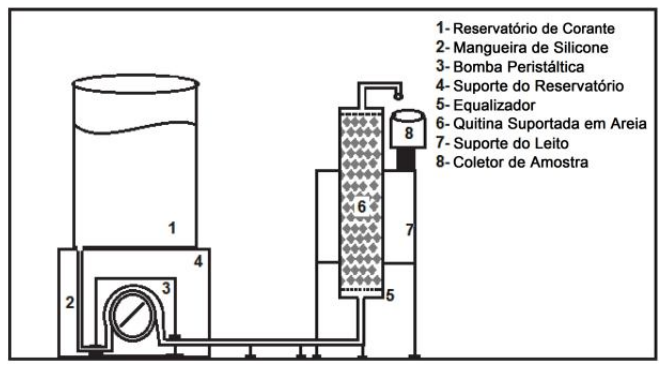

Fonte: Autor (2015).

A coluna de leito de fixo, com $2.5 \mathrm{~cm}$ de diâmetro interno e $25.0 \mathrm{~cm}$ de altura, foi 
preenchida com $180 \mathrm{~g}$ de areia (suporte inerte) e $5 \mathrm{~g}$ de quitina-SMU.

Um efluente sintético, contendo Azul de Metileno, com concentração de $50 \mathrm{mg} \mathrm{L}^{-1} \mathrm{e}$ $\mathrm{pH} 10$, foi bombeado através do leito, com

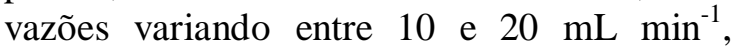
utilizando uma bomba peristáltica (Master Flex, 07553-75).

As amostras de efluentes foram coletadas no topo da coluna em intervalos de tempo regulares. A concentração de corante, remanescente em solução foi analisada via espectrofotometria, com comprimento de onda de $664 \mathrm{~nm}$.

\subsection{Análise de Dados da Coluna}

A análise de dados da coluna foi feita de modo a obter o tempo de ruptura $\left(t_{b}\right)$, tempo de exaustão $\left(\mathrm{t}_{\mathrm{e}}\right)$, comprimento da zona de transferência $\left(Z_{m}\right)$, volume de efluente tratado $\left(\mathrm{V}_{\mathrm{eff}}\right)$, capacidade máxima da coluna $\left(\mathrm{q}_{\mathrm{eq}}\right)$ e percentual de remoção $(\mathrm{R})$, para cada curva (GEANKOPLIS, 1998). O tempo de ruptura $\left(\mathrm{t}_{\mathrm{b}}, \mathrm{min}\right)$ foi considerado quando a concentração de MB na saída da coluna alcançou $5 \%$ da concentração de entrada. $\mathrm{O}$ tempo de exaustão, quando a concentração de MB atingiu 95\% da concentração inicial (SUZUKI, 1990). A $Z_{\mathrm{m}}(\mathrm{cm})$ reflete o menor comprimento possível do leito de adsorção a fim de que se obtenha o tempo de ruptura em $\mathrm{t}=0$ (Suzuki, 1990).

As curvas de ruptura foram graficadas em termos da concentração normalizada, $\mathrm{C}_{\mathrm{t}} / \mathrm{C}_{0}$, que é definida como a taxa de concentração de corante efluente da coluna, $\mathrm{C}_{\mathrm{t}}$ $\left(\mathrm{mg} \mathrm{L}^{-1}\right)$ pela concentração de corante na corrente de entrada, $\mathrm{C}_{0}\left(\mathrm{mg} \mathrm{L}^{-1}\right)$ como uma função do tempo. $\mathrm{O}$ cálculo do comprimento da zona de transferência foi realizado utilizando a Equação 1:

$\mathrm{Z}_{\mathrm{m}}=\mathrm{Z}\left(1-\frac{\mathrm{t}_{\mathrm{r}}}{\mathrm{t}_{\mathrm{e}}}\right)$
$\mathrm{O}$ volume de efluente tratado, $\mathrm{V}_{\text {eff }}$ (mL), é dado pela Equação 2:

$\mathrm{V}_{\mathrm{eff}}=\mathrm{Qt}_{\text {total }}$

onde, Q é vazão $\left(\mathrm{mL} \min ^{-1}\right)$ e $\mathrm{t}_{\text {total }}$ é o tempo total de operação (min).

A capacidade máxima da coluna, $\mathrm{q}_{\mathrm{eq}}$ $\left(\mathrm{mg} \mathrm{g}^{-1}\right)$ é dada pela Equação 3:

$\mathrm{q}_{\mathrm{eq}}=\frac{\frac{\mathrm{QC}_{0}}{1000} \int_{0}^{\mathrm{t}_{\text {total }}} \mathrm{Ydt}}{\mathrm{m}}$

onde, $\mathrm{C}_{0}$ é a concentração inicial de $\mathrm{MB}(\mathrm{mg}$ $\mathrm{L}^{-1}$ ) e $\mathrm{m}$ é a massa (g) de quitina-SMU contida no leito. A integral, na Equação 3 é a área acima da curva de ruptura, de $\mathrm{C}_{\mathrm{t}} / \mathrm{C}_{0}=0$ até $\mathrm{C}_{\mathrm{t}} / \mathrm{C}_{0}=1$.

O percentual de remoção (R) foi calculado pela Equação 4:

$\mathrm{R}=\frac{\int_{0}^{\mathrm{t}_{\text {toal }}}\left(1-\frac{\mathrm{C}_{\mathrm{t}}}{\mathrm{C}_{0}}\right) d t}{\mathrm{t}_{\text {total }}} 100$

\subsection{Aplicação de Modelos Dinâmicos}

Os dados experimentais foram ajustados, na melhor condição, a três modelos dinâmicos comuns: BDST (Equação 5), Thomas (Equação 6) e Yoon-Nelson (Equação 7).

$$
\begin{aligned}
& \frac{\mathrm{C}_{0}}{\mathrm{C}_{\mathrm{t}}}=1+\exp \left(\frac{\mathrm{KN}_{0} \mathrm{~h}}{\mathrm{u}}-\mathrm{KC}_{0} \mathrm{t}\right) \\
& \frac{\mathrm{C}_{0}}{\mathrm{C}_{\mathrm{t}}}=1+\exp \left(\frac{\mathrm{k}_{\mathrm{Th}} \mathrm{q}_{\mathrm{e}} \mathrm{m}}{\mathrm{Q}}-\mathrm{k}_{\mathrm{Th}} \mathrm{C}_{0} \mathrm{t}\right) \\
& \frac{\mathrm{C}_{0}}{\mathrm{C}_{\mathrm{t}}}=1+\exp \left(\tau \mathrm{k}_{\mathrm{YN}}-\mathrm{k}_{\mathrm{YN}} \mathrm{t}\right)
\end{aligned}
$$

onde, $\mathrm{Z}$ é o comprimento do leito. 
onde, $\mathrm{K}$ é a constante de adsorção $\left(\mathrm{mL} \mathrm{mg}^{-1}\right.$ $\left.\min ^{-1}\right), \mathrm{N}_{0}$ é a capacidade de adsorção ( $\mathrm{mg} \mathrm{L}^{-}$ $\left.{ }^{1}\right)$, h é a altura do leito fixo $(\mathrm{cm})$, u é a velocidade linear $\left(\mathrm{cm} \min ^{-1}\right), K_{T h}$ é a constante do modelo de Thomas $\left(\mathrm{mL} \mathrm{mg}^{-1}\right.$ $\left.\min ^{-1}\right), \mathrm{q}_{\mathrm{eq}}$ é a capacidade de adsorção no equilíbrio no modelo de Thomas ( $\left.\mathrm{mg} \mathrm{g}^{-1}\right)$, $\mathrm{m}$ é a massa de adsorvente na coluna (g), $\mathrm{K}_{\mathrm{YN}}$ é a constante do modelo de Yoon-Nelson $\left(\mathrm{min}^{-1}\right)$ e $\tau$ é o tempo requerido para que o efluente da coluna atinja $50 \%$ da concentração inicial de adsorbato do modelo de Yoon-Nelson (min).

Os parâmetros dinâmicos foram determinados através de regressão não-linear utilizando o software Statistic 9.1 (StatSoft Inc.). A qualidade do ajuste foi determinada utilizando o coeficiente de determinação $\left(\mathrm{R}^{2}\right)$ e soma de erros quadrados (SSE) (ELKHAIARY e MALASH, 2011).

\subsection{Testes de Eluição}

Os testes de eluição foram realizados a fim de verificar a capacidade de regeneração do leito e a possibilidade de reuso para consecutivos ciclos. Após a adsorção, a quitina-SMU/areia carregadas de $\mathrm{MB}$, foram removidas da coluna, secas a $60{ }^{\circ} \mathrm{C}$, durante $24 \mathrm{~h}$ e, acomodadas novamente no leito. Os experimentos de eluição foram realizados utilizando o mesmo sistema apresentado na Figura 1. Solução de $\mathrm{HCl} 0.3 \mathrm{~mol} \mathrm{~L}^{-1}$, foi utilizada como eluente (DOTTO et al., 2015), com vazão de $20 \mathrm{~mL} \mathrm{~min}^{-1}$. A concentração de $\mathrm{MB}$, no topo da coluna, foi determinada de acordo com a Seção 2.2.

\section{RESULTADOS E DISUSSÃO}

\subsection{Características da Quitina Modificada}

A quitina-SMU foi caracterizada através de MEV, BET, FT-IR e XRD. As imagens do MEV para quitina sem tratamento e para a quitina-SMU estão apresentadas na Figura 2.
Figura 2 - Imagens do MEV (a) para quitina sem tratamento e (b) para a quitina-SMU
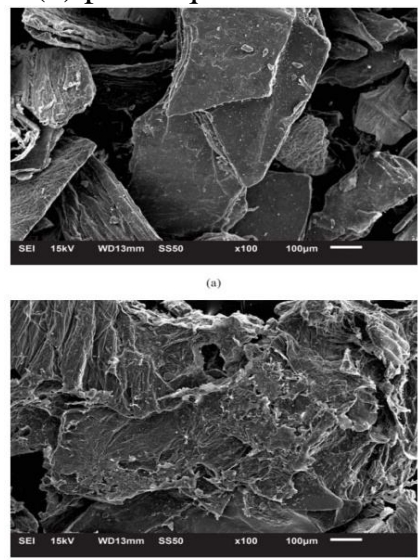

Fonte: Autor (2015).

Uma superfície rígida e lisa, sem poros visíveis ou cavidades pode ser observada na Figura 2(a). Na Figura 2(b), a quitina-SMU apresentou superfície áspera, com protuberâncias e algumas cavidades. A área superficial específica da quitina in natura e da tratada foram, respectivamente, 2.5 e $50.8 \mathrm{~m}^{2}$ $\mathrm{g}^{-1}$. As bandas do FT-IR e as respectivas atribuições para a quitina-SMU estão apresentadas na Tabela 1 (as mesmas bandas foram encontradas para a quitina sem tratamento).

Tabela 1 - Bandas do FT-IR e atribuições para quitina-SMU.

\begin{tabular}{|c|c|}
\hline Bandas $\left(\mathrm{cm}^{-1}\right)$ & Atribuições \\
\hline 3350 & $\begin{array}{c}\text { Estiramentos de } \mathrm{O}-\mathrm{H} \\
\text { e } \mathrm{N}-\mathrm{H}\end{array}$ \\
\hline 3000 & Estiramento de $\mathrm{CH}_{2}$ \\
\hline 2800 & Estiramento de $\mathrm{CH}_{3}$ \\
\hline 1652 & $\begin{array}{l}\text { Estiramento de } \mathrm{C}=\mathrm{O} \\
\text { de amida secundária }\end{array}$ \\
\hline 1551 & $\begin{array}{c}\text { Deformação de } \mathrm{N}-\mathrm{H} \\
\text { e estiramento de } \mathrm{C}-\mathrm{N} \\
\text { de amida primária }\end{array}$ \\
\hline 1018 & $\begin{array}{l}\text { Estiramento } \\
\text { assimétrico de } \mathrm{C}-\mathrm{O} \\
\text { em anel }\end{array}$ \\
\hline 745 & $\begin{array}{l}\text { Deformação de }-\mathrm{NH} \\
\text { for a do plano }\end{array}$ \\
\hline
\end{tabular}


Tanto para a quitina in natura, como para a quitina-SMU, o grau de desacetilação foi $45 \pm 1 \%$. O índice de cristalinidade foi de $85 \%$ para a quitina in natura e de $63 \%$ para a quitina-SMU. Estes resultados estão de acordo com trabalhos apresentados anteriormente (DOTTO et al., 2015) e confirmam que a quitina-SMU tem características mais adequadas para os processos de adsorção quando comparada à quitina sem tratamento.

\subsection{Interpretação das curvas de ruptura}

As curvas de ruptura foram construídas em diferentes vazões $\left(10,15\right.$, e $\left.20 \mathrm{~mL} \mathrm{~min}^{-1}\right)$ e concentração inicial de $\mathrm{MB}$ de $50 \mathrm{mg} \mathrm{L}^{-1}$ e estão apresentadas na Figura 3.

Figura 3 - Curvas de ruptura

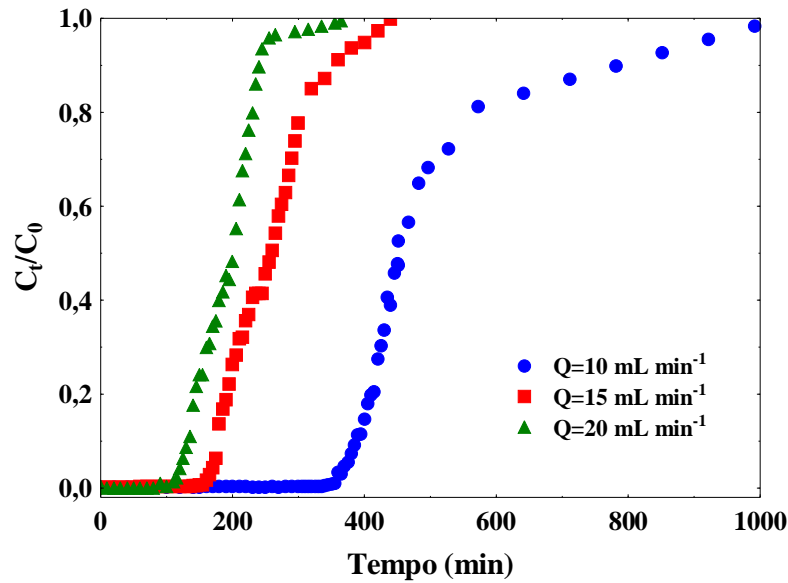

Fonte: Autor (2015).
Os resultados em relação ao tempo de ruptura $\left(\mathrm{t}_{\mathrm{b}}\right)$, tempo de exaustão $\left(\mathrm{t}_{\mathrm{e}}\right)$, comprimento da zona de transferência $\left(\mathrm{Z}_{\mathrm{m}}\right)$, volume de efluente tratado $\left(\mathrm{V}_{\text {eff }}\right)$, capacidade máxima da coluna $\left(\mathrm{q}_{\mathrm{eq}}\right)$ e o percentual de remoção $(\mathrm{R})$ estão apresentados na Tabela 2.

Analisando os dados contidos na Tabela 2 constatou-se que da coluna foi, em geral, melhor a baixas vazões. Três fatos podem ser analisados na Figura 3 e na Tabela 2: 1) a diminuição da vazão implicou em tempos de ruptura e exaustão mais longos; 2) os mais altos valores para $\mathrm{q}_{\mathrm{eq}} \mathrm{e} \mathrm{V}_{\text {eff }}$ foram obtidos quando e a vazão foi de $10 \mathrm{~mL} \min ^{-1}$; 3) apesar do melhor desempenho do leito ter sido com vazão de $10 \mathrm{~mL} \mathrm{~min}^{-1}$, o maior valor de $R$ não foi encontrado nesta mesma condição. A primeira observação deve-se a que baixas vazões causam aumento do tempo de residência no leito, permitindo maior interação entre a quitina-SMU e o MB. Quando a vazão é aumentada, o tempo de residência não é suficiente para garantir a difusão de MB na estrutura da quitina-SMU. $\mathrm{O}$ segundo fato ocorre porque em baixas vazões, foram obtidas curvas alongadas, indicando que volumes maiores de efluentes podem ser tratados. Como consequência, a área acima da curva foi maior, melhorando o desempenho do leito. O terceiro fato ocorreu porque o grande comprimento da zona de transferência, encontrado no experimento 1 , fez com que a corrente de saída do leito tivesse uma maior concentração de $\mathrm{MB}$, durante um intervalo de tempo mais longo.

Tabela 2 - Condições experimentais e os resultados da adsorção de MB em quitina-SMU suportada por areia.

\begin{tabular}{ccccccccc}
\hline Exp. & $\begin{array}{c}\text { Vazão, } \mathrm{Q} \\
\left(\mathrm{mL} \mathrm{min}^{-1}\right)\end{array}$ & $\begin{array}{c}\mathrm{C}_{0} \\
\left(\mathrm{mg} \mathrm{L}^{-1}\right)\end{array}$ & $\begin{array}{c}\mathrm{t}_{\mathrm{b}} \\
(\mathrm{min}) *\end{array}$ & $\begin{array}{c}\mathrm{t}_{\mathrm{e}} \\
(\mathrm{min}) *\end{array}$ & $\mathrm{Z}_{\mathrm{m}}(\mathrm{cm})^{*}$ & $\begin{array}{c}\mathrm{V}_{\text {eff }} \\
(\mathrm{mL})^{*}\end{array}$ & $\begin{array}{c}\mathrm{q}_{\mathrm{eq}} \\
\left(\mathrm{mg} \mathrm{g}^{-1}\right)^{*}\end{array}$ & $\mathrm{R}(\%)^{*}$ \\
\hline 1 & 10 & 50 & 370 & 922 & 15.0 & 9920 & 51.8 & 51.5 \\
2 & 15 & 50 & 171 & 400 & 14.3 & 6600 & 38.1 & 58.7 \\
3 & 20 & 50 & 122 & 250 & 12.9 & 7500 & 38.7 & 51.3 \\
\hline
\end{tabular}




\subsection{Modelos Dinâmicos}

Os resultados obtidos a partir do ajuste dos dados experimentais aos modelos dinâmicos estão apresentados na Figura 4 e na Tabela 3.

Figura 4 - Gráfico dos dados experimentais ajustados aos modelos dinâmicos

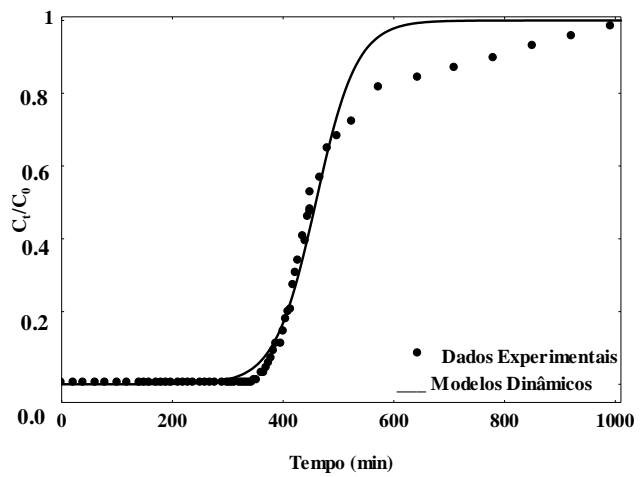

Fonte: Autor (2015).

Tabela 3 - Parâmetros dinâmicos para adsorção de MB de acordo com os modelos BDST, Thomas e Yoon-Nelson (na melhor condição).

\begin{tabular}{cc}
\hline \multicolumn{3}{c}{ Modelo BDST } \\
\hline $\mathrm{K}\left(\mathrm{ml} \mathrm{mg}^{-1} \mathrm{~min}^{-1}\right)$ & 0.54 \\
$\mathrm{~N}_{0}\left(\mathrm{mg} \mathrm{L}^{-1}\right)$ & 1870 \\
$\mathrm{R}^{2}$ & 0.9781 \\
$\mathrm{SSE}$ & 0.1361 \\
\hline \multicolumn{2}{c}{ Modelo de Thomas } \\
\hline $\mathrm{K}_{\mathrm{Th}}\left(\mathrm{mL} \mathrm{mg}^{-1} \mathrm{~min}^{-1}\right)$ & 0.54 \\
$\mathrm{q}_{\mathrm{eq}}\left(\mathrm{mg} \mathrm{g}^{-1}\right)$ & 45.9 \\
$\mathrm{q}_{\mathrm{eq}}(\mathrm{exp})\left(\mathrm{mg} \mathrm{g}^{-1}\right)$ & 51.8 \\
$\mathrm{R}^{2}$ & 0.9781 \\
$\mathrm{SSE}$ & 0.1361 \\
\hline \multicolumn{2}{c}{ Modelo de Yoon-Nelson } \\
\hline $\mathrm{K}_{\mathrm{YN}}\left(\mathrm{min}^{-1}\right)$ & 0.027 \\
$\tau(\mathrm{min})$ & 458 \\
$\tau_{\text {(exp) }}(\mathrm{min})$ & 452 \\
$\mathrm{R}^{2}$ & 0.9781 \\
$\mathrm{SSE}$ & 0.1361 \\
\hline
\end{tabular}

Tendo como base os altos valores de $\mathrm{R}^{2}$ $\left(\mathrm{R}^{2}>0.97\right)$ e os baixos valores de SSE (SSE<0.14), pode-se afirmar que os três modelos citados foram adequados para representar a curva experimental de ruptura (na melhor condição).

\subsection{Regeneração da Coluna}

A curva de eluição após o quarto ciclo de adsorção, está apresentada na Figura 5.

Figura 5 - Curva de eluição após o quarto ciclo de adsorção

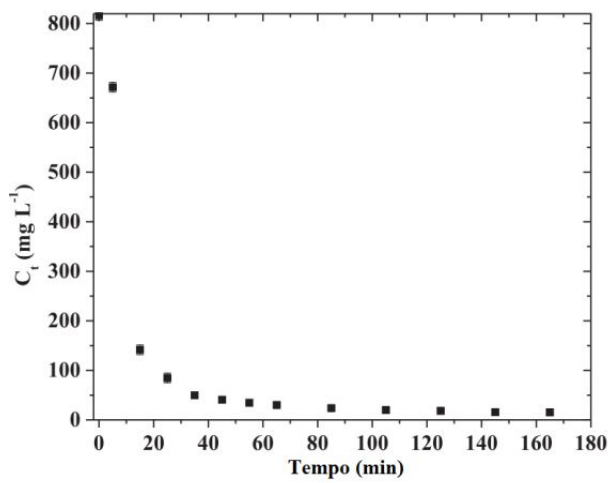

Fonte: Autor (2015).

Foi observado que, após o quarto ciclo, todo corante foi removido do leito em até 150min. O desempenho do leito em termos de $t_{b}, t_{e}, Z_{m}, V_{\text {eff }}, q_{e q}$ e R foi mantido depois de cinco ciclos de adsorção-eluição. Após os cinco ciclos, o desempenho foi fortemente comprometido. Em geral, os resultados confirmaram a viabilidade da técnica da adsorção de MB em leito fixo utilizando quitina-SMU suportada em areia.

\section{CONCLUSÃO}

A viabilidade da técnica da adsorção, em leito fixo de MB em quitina-SMU foi avaliada visando um futuro aumento de escala. O melhor desempenho do leito foi obtido com vazão de $10 \mathrm{~mL} \mathrm{~min}{ }^{-1}$. O tempo de ruptura foi de 370 min, a capacidade máxima da coluna foi $51.8 \mathrm{mg} \mathrm{g}^{-1} \mathrm{e}$ o percentual de remoção foi de $51.5 \%$. No total, foram tratados $9920 \mathrm{~mL}$ de efluente sintético. Nas condições mais apropriadas do leito, os modelos BDST, Thomas e Yoon-Nelson 
foram adequados para obter os parâmetros do leito, como $\tau$ e $\mathrm{q}_{\text {eq. }}$. $\mathrm{O}$ desempenho do leito foi mantido após cinco ciclos de adsorçãoeluição.

\section{NOMENCLATURA}

$\mathrm{C}_{0} \quad$ concentração inicial de $\mathrm{MB}, \mathrm{mg} \mathrm{L}^{-1}$.

$\mathrm{C}_{\mathrm{t}}$ concentração de MB na saída da coluna, $\operatorname{mg~L^{-1}}$.

h profundidade do leito fixo, $\mathrm{cm}$.

$\mathrm{K}$ constante de adsorção, $\mathrm{mL} \mathrm{mg}^{-1} \mathrm{~min}^{-1}$.

$\mathrm{k}_{\mathrm{Th}}, \quad$ constante do modelo de Thomas, $\mathrm{mL} \mathrm{mg}^{-1}$ $\min ^{-1}$.

$\mathrm{k}_{\mathrm{YN}}$ constante do modelo de Yoon-Nelson, $\min ^{-1}$.

m massa de adsorvente na coluna, g.

$\mathrm{N}_{0} \quad$ capacidade de adsorção, $\mathrm{mg} \mathrm{L}^{-1}$.

Q vazão volumétrica, $\mathrm{mL} \min ^{-1}$.

R percentual de remoção de $\mathrm{MB}, \%$.

$\mathrm{R}^{2}$ coeficiente de determinação, adimensional.

$\mathrm{t}$ tempo, min.

$t_{b} \quad$ tempo de breakthrough, min.

$t_{\mathrm{e}} \quad$ tempo de exaustão, min.

$\mathrm{t}_{\text {total }} \quad$ tempo total de operação, min.

u taxa de fluxo linear, $\mathrm{cm} \mathrm{min}^{-1}$.

$\mathrm{V}_{\text {eff }} \quad$ volume de efluente, $\mathrm{mL}$.

$\mathrm{Z} \quad$ comprimento do leito, $\mathrm{cm}$.

$\mathrm{Z}_{\mathrm{m}} \quad$ comprimento da zona de transferência de massa, $\mathrm{cm}$.
Parâmetro do modelo de Yoon-Nelson, min.

\section{REFERÊNCIAS}

AL-SAGHEER, F. A.; AL-SUGHAYER, M. A.; MUSLIM, S.; ELSABEE, M. Z. Extraction and characterization of chitin and chitosan from marine sources in Arabian Gulf. V.77, p. 410-419, 2009.

BHATNAGAR, A.; SILLANPÄÄ, M. Applications of chitin derivates for the detoxification of water and wastewater. V. 152, p. 26-38, 2009.

BREITBACH, M.; BATHEN, D.; Influence of ultrasound on adsorption processes. V.8, p. 277-283, 2001.

BRUNAUER, S.; EMMETT, P. H.; TELLER, E. Adsorption of gases in multimolecular layers. V.60, p. 309318,1938 .

DOTTO, G. L.; SANTOS, J. M. N.; RODRIGUES, I.L.; ROSA, R.; PAVAN, F.A.; LIMA, E.C. Adsorption of Methylene Blue by ultrasonic surface modified chitin. V. 446, p.133-140, 2015.

DOTTO, G.L.; PINTO, L.A.A. Adsorption of food dyes acid blue 9 and food yellow 3 onto chitosan: stirring rate effect in kinetics and mechanism. V. 187, p.164170, 2011.

DOTTO, G.L.; VIEIRA, M.L.G.; PINTO, L.A.A. Kinetics and mechanism of tartrazine adsorption onto chitin and chitosan. V.51, p.6862-6868, 2012.

EL-KHAIARY, M.I.; Malash, G.F. Common data analysis errors in batch adsorption studies. V.105, p.314-320, 2011. 
ESQUERDO, V.M.; CADAVAL, T.R.S. Jr.; DOTTO, G.L.; PINTO, L. A. A. Chitosan scaffold as an alternative adsorbent for the removal of hazardous food dyes from aqueous solutions. V. 424, p. 7-15, 2014.

GEANKOPLIS, C. J. Procesos de transporte y operaciones unitárias. Compañía editorial continental, 1998.

GOLDSTEIN, J. I.; NEWBURY, D. E.; ECHIL, P.; JOY, D. C.; ROMIG, A.D. Jr.; LYMAN, C. E.; FIORI, C.; LIFSHIN, E. Scanning electron microscopy and $\mathrm{X}$-ray microanalysis. 1992.

KAO, P.M.; CHEN, C.I.; HUANG, S.C.; LIN, K.M.; CHANG, Y.C.; LIU, Y.C. Preparation of fermentation-processed chitin and its application in chitinase affinity adsorption. V.44, p. 343-348, 2009.

MOURA, C.; MUSZINSKI, P.; SCHIMDT, C.; ALMEIDA, J.; PINTO, L. Quitina e quitosana produzidas a partir de resíduos de camarão e siri: avaliação do processo em escala piloto. V.16, p. 37-45, 2006.

SILVERSTEIN, R. M.; WEBSTER, F. X.; KIEMLE, $\quad$ D. J. Spectrometric identification of organic compounds, 2007.

SUZUKI, M. Adsorption Engineering, 1990.

VIEIRA, M. L. G.; ESQUERDO, V. M.; NOBRE, L. R.; DOTTO, G. L.; PINTO, L.A.A. Glass beads coated with chitosan for the food azo dyes adsorption in a fixed bed column. V. 20, p. 3387-3393, 2014.

WALKER, G.M.; HANSEN, L.; HANA, J.A.; ALLEN, S.J. Kinetics of a reactive dye adsorption onto dolomitic sorbents. V.37, p. 2081-2089, 2003.
YIN, C.Y.; AROUA, M.K.; DAUD, W.M.A.W. Fixed-bed adsorption of metal ions from aqueous solution on polyethyleneimine-impregnated palm shell activated carbon. V.148, p. 8-14, 2009.

ZOLLINGER, H. Color ChemistrySynthesis, Properties, and Applications of Organic Dyes and Pigments. Vol 67, p. 386386, 1992. 